\title{
EQUILIBRIUM OF THE RETURN-CURRENT SHEET AND STRUCTURE OF THE PULSAR MAGNETOSPHERE
}

\author{
YU. E. LYUBARSKII \\ Institute of Radio Astronomy
}

Pulsars are generally identified with rotating, magnetized neutron stars. According to Goldreich and Julian (1969), an induced electric field creates the electric current which flows out of the magnetic poles and fills the magnetosphere with plasma. The current flows along the open magnetic field lines and returns along the boundary between the closed and open parts of the magnetosphere (Scharlemann and Wagoner 1973). The boundary shape is determined by the equilibrium condition for the current sheet.

The mechanical equilibrium of the current sheet is controlled by the electrostatic and Lorentz forces. I adopt the local set of coordinates in which the $z$ axis is orthogonal to the sheet; then

$$
\rho E_{z}+\left(j_{x} B_{y}-j_{y} B_{x}\right) / c=0 .
$$

Here $\rho$ and $j$ are the charge and current densities. The electric and magnetic fields satisfy the Maxwell equations

$$
\begin{gathered}
d E_{z} / d z=4 \pi \rho \\
d B_{y} / d z=-4 \pi j_{x} / c \\
d B_{x} / d z=4 \pi j_{y} / c
\end{gathered}
$$

Combination of eqs.(1) to (4) yields the equilibrium condition

$$
\frac{d}{d z}\left(B^{2}-E^{2}\right)=0
$$

and the coupling between the electromagnetic fields in open and closed parts of the magnetosphere

$$
B_{0}^{2}-E_{0}^{2}=B_{c}^{2}-E_{c}^{2}
$$

The structure of the magnetosphere is described by the stream function $f$. In the closed part of the magnetosphere the pulsar equation in the dimensionless cylindrical coordinates $(z \Rightarrow c z / \Omega, r \Rightarrow$ $\mathrm{cr} / \Omega$ ) takes the form (Michel 1973)

$$
\Delta f\left(1-r^{2}\right)-\frac{2}{r} \frac{\partial f}{\partial r}=0 .
$$

In the open part of the magnetosphere the pulsar equation can be reduced to a linear form (Scharlemann and Wagoner 1973, Beskin, Gurevich, and Istomin 1983b);

$$
\Delta F\left(1-x^{2}\right)-\frac{2}{x} \frac{\partial F}{\partial x}+\alpha F=0,
$$

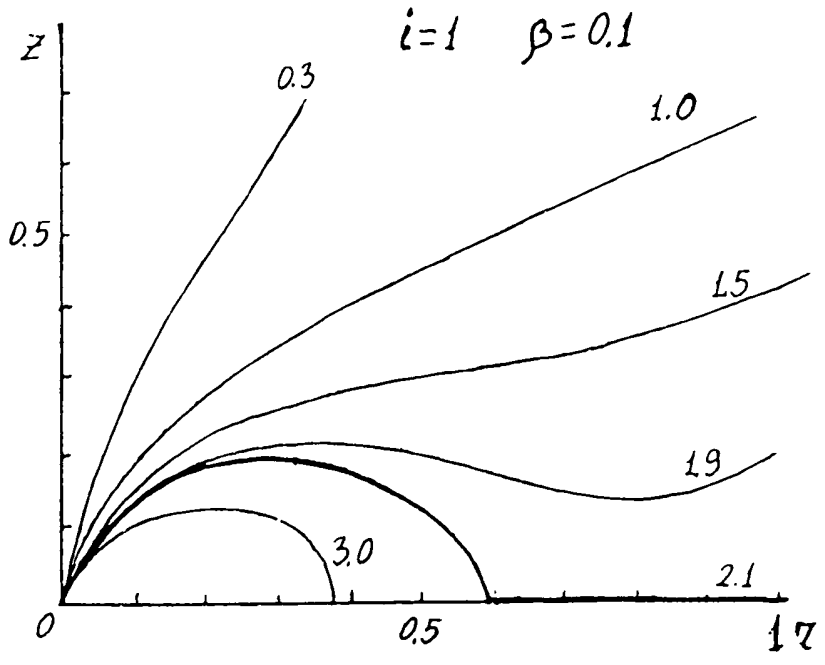

Figure 1a

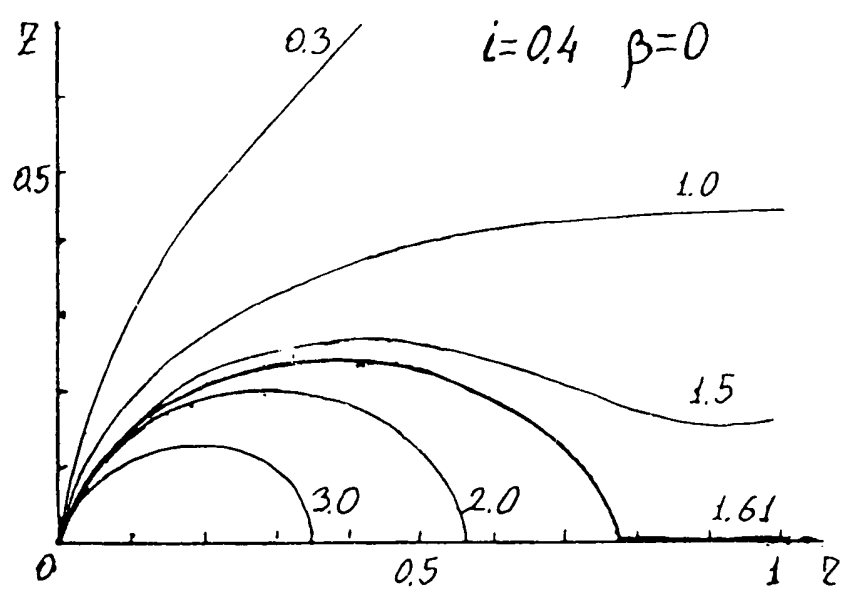

Figure 1 b

where $F$ is the stream function in this part of the magnetosphere

$$
\begin{gathered}
x=r(1-\beta) ; \\
z_{1}=z(1-\beta) ; \\
\alpha=i^{2} /(1-\beta)^{2} ;
\end{gathered}
$$

$i$ and $b$ are dimensionless constants, determining the longitudinal current and the potential drop between the neutron star and the magnetosphere. Matching the solutions $F$ and $f$ on the separatrix

$$
F(r, z)=f(r, z)=f^{*}
$$


should be performed in accordance with eq.(6). In terms of the stream functions this condition can be written in the form

$\left(1-r^{2}\right)[\nabla f]_{f=f^{*}}^{2}=i^{2} f^{* 2}+\left[1-(1-\beta)^{2} r^{2}\right][\nabla f]_{F=f^{*}}^{2}$

The structure of the magnetosphere is determined by the shape of the separatrix. It can be established qualitatively from an analysis of the equilibrium condition. There are two branches of the separatrix; the first branch goes around the closed part of the magnetosphere but the second one goes to infinity. There is a break at the point where both branches connect; therefore at this point we have $\nabla F=0$. According to eq.(11), $\nabla f$ should be nonzero at this point, hence there is no cusp in the closed part of the magnetosphere, contrary to the result of Beskin et al. (1983).

A numerical solution of eqs.(10) and (11) is shown in figure 1 (Lyubarskii 1990). The entire closed part of the magnetosphere is inside the light cylinder, but this part of the magnetosphere grows when the longitudinal current decreases. This behavior is consistent with the fact, according to Michel (1973), that the closed part of the currentfree magnetosphere extends up to the light cylinder, and $f^{*}=1.59$ in this case. The solution obtained does not require any additional constraint on parameters such as the "matching condition" of Beskin et al. (1983) wherein they connect the longitudinal current and the potential drop between the neutron star and the open part of the magnetosphere. 